Invited commentary on:

\title{
Variations in the costs of child and adolescent
} psychiatric in-patient units $^{\dagger}$

\section{EXPLAINING AND INTERPRETING VARIATIONS IN COSTS}

The great majority of young people with mental health problems never need admission to hospital. A small number, however, require some kind of in-patient provision. In comparison with North America and many parts of Europe, such provision has now become relatively uncommon in the UK. Although the remaining services are widely believed to be costly, there are no national data collections that provide accurate economic data. To begin to meet this need, the Department of Health commissioned a national study to explore the costs of these units, which ran in parallel with the National In-patient Child and Adolescent Psychiatry Study (NICAPS).

The paper by Beecham et al (2003, this issue) analyses variations in costs between psychiatric in-patient units for children and adolescents. The main finding is that costs vary fourfold. The main conclusion is that this variation should be used to help commissioners and providers as they develop commissioning strategies.

The methods used to describe and analyse the costs of children's mental health services are clearly at an early stage. The questionnaire used by Beecham et al is of unknown reliability, and the validity of using cost estimates obtained from hospital personnel was not established. Moreover, some of the assumptions behind the statistical analyses are debatable, such as the decision to exclude staffing levels from all analyses of the influences on cost variation.

Nevertheless, economic research such as this can have important implications for the planning of services. Politicians and health service managers are understandably concerned to know whether economies can be achieved through more

†See pp. 220-225, this issue. rational use of what are often called 'highcost low-volume' services. They will look at the wide variation in costs between child psychiatric in-patient services and ask whether these can be justified in terms of the needs of patients. If not, then they may argue that pressure should be brought to bear on high-cost units to reduce their costs.

\section{Explaining variation}

At first sight then, the finding that the costs of child psychiatric in-patient services vary from $£ 91$ to $£ 380$ per patient per day might be interpreted as indicating inefficient use of resources. However, this would only be the case if costs were not predictable or were predicted by factors unrelated to patient care. In the NICAPS the costs were not random. On the contrary, multivariate statistical analyses showed that more than $50 \%$ of the costs could be predicted (and this proportion was probably an underestimate because some of the reported variation was undoubtedly due to statistical chance).

The costs of the services that took part in the NICAPS were not only predictable in a statistical sense but also could be related meaningfully to indices of the patients' quality of care and his or her health needs. For example, costs were higher in units that were more spacious. Costs were lower in units with more beds and in those outside of London where staff costs were presumably less. Costs were also related to measures of clinical need, such as the presence of a mood disorder or learning disability.

\section{Does cost variation influence clinical outcomes?}

Providers of in-patient services can therefore take some comfort from this economic analysis. Differences in the costs of in-patient units are not random. They are linked to important differences in the quality of service provision and in the patients' needs. But do these differences in costs tell us anything about the effectiveness of services? For example, do patients in smaller, better-staffed units do better than patients in larger units with a lower staff/patient ratio? Information on cost per se is not sufficient to answer these questions. We do not know what is a desirable cost for an in-patient admission. The cost comparisons provided here do not, for example, enable the in-patient costs of rare paediatric conditions to be compared with morbidity and mortality to anorexia nervosa or schizophrenia. In order to address this issue it is essential to know much more about the relationship between costs and outcomes. This requires research on the clinical outcomes and satisfaction of comparable patients who attend highand low-cost in-patient services. To date, research on this issue in the UK has been very limited.

\section{Implications for commissioners}

In the meantime, some health service planners may make the mistaken assumption that variation in costs is a sign of inefficiency. They may infer that children should be placed in larger, more crowded units, or that units in expensive locations such as London should be closed in favour of units in less-expensive settings. Although this argument may be tempting from the point of view of costs, and is difficult to resist given the very limited evidence base on outcomes, there are good reasons for rejecting it.

First, in the absence of central planning, in-patient units have developed in an ad hoc fashion, with individual aims, functions and client groups. With no model of a standard generic in-patient unit, this analysis does not compare like with like and therefore variation is surely to be expected. Second, child care policy and research over the past 30 years have consistently stressed that when it is necessary for children to be placed in residential facilities, such as hospitals, their care should be personalised and there should be close links with the family. This is likely to be very difficult to achieve if children are placed in large inpatient units many miles from their homes. Third, although spaciousness within a unit mistakenly could be considered a 'hotel' 
issue, space in a low-stimulus environment is believed to contribute to clinical outcomes of, for example, psychotic disorder.

\section{Conclusions}

To conclude, health economics analyses are a welcome contribution to considering the effectiveness of services, but their findings should be interpreted with caution. The debate about the best ways to provide services for children with severe mental health problems is not just economic, but also involves clinical and humanitarian issues. It is to be hoped that the initiatives of health economists will motivate clinicians to improve the quality of their outcome data in order to provide both service users and commissioners with quality information to support their decision-making.

Beecham, J., Chisholm, D., O'Herlihy, A., et al (2003) Variations in the costs of child and adolescent psychiatric in-patient units. British Journal of Psychiatry, 183, 220-225.

\section{Declaration of interest}

None.

Richard Harrington FRCPsych, Department

of Child and Adolescent Psychiatry, Royal

Manchester Children's Hospital, Manchester

M27 4HA, UK

Simon Gowers FRCPsych, Department of Child and Adolescent Psychiatry, University of Liverpool, UK 